\title{
A Study of Strategies for Chinese-English Translation of Technical English Vocabulary in Cognitive Context
}

\author{
Yan Jiang \\ NanChang Institute of Technology,Nanchang,330099
}

\begin{abstract}
Keyword: Technical english vocabulary; Conceptual integration theory; Chinese-English translation strategies
\end{abstract}

\begin{abstract}
With the continuous development of the global economy, politics and culture, the relationship between science and technology and human cognition is becoming closer and closer. Therefore, more and more attention has been paid to the studies of translating technical English. As an important part of technical English, the translation of technical English vocabulary into Chinese has attracted much attention. From the new perspective of cognition and taking the theory of conceptual integration as the core theory, this paper uses the research methods of qualitative analysis and quantitative analysis to study the technical English vocabulary and its strategies of Chinese translation. Based on the theory of conceptual integration, this paper attempts to propose that the cognition model should be integrated properly and rationally using the concept of language translation in the translation of technical English vocabulary. By studying the translation of technical English vocabulary, international exchanges and cooperation in science and technology between different countries are strengthened, which helps translators improve the quality of translation and achieve effective translation.
\end{abstract}

\section{Introduction}

Traditional translation theories have limited the translation strategies of technical English vocabulary. Attempts are made to apply conceptual integration theory to the translation of technical English vocabulary from a cognitive perspective and further explore the definition, characteristics and classification of technical English words as well as the source, mapping and composition of its meaning. This paper conducts qualitative research and quantitative analysis of the various translation strategies of technical English vocabulary and obtains the empirical research findings based on the relevant data results. This reveals and establishes the system of Chinese translation strategies and methods of technical English vocabulary. Based on the cognition of technical English vocabulary, a series of effective translation strategies are put forward comprehensively and systematically, including literal translation, free translation, transliteration, morphological translation, classified translation, word class conversion translation, complementary translation, omission, replacement translation, logical phrase translation and nominalization translation, and in-depth analysis is carried out to study the relationship between different translation strategies and related corpus data.

\section{Technical English}

After the end of World War II, the role of English in international politics, economy, culture and science and technology became more and more obvious. As the number of English literatures increased in geometric progression, English became an international language. In China's current science and technology works, English is the preferred language. The ultimate goal in learning technical English is to use it as a powerful tool to understand the development trend of science and technology in the world and to exchange ideas with international academic communities. Therefore, the application of technical English is reflected in its communicative function.

Communicative function can be achieved by listening, speaking, reading and writing. Listening and speaking are the most basic ways for people to communicate. Reading refers to reading a variety of science and technology articles, including popular science articles and special science articles. Specialized technical articles include digests, dissertations, standards, patents (brochures), minutes, lab 
reports, government research reports, technical contracts, letters of intent, product samples, and brochures. Writing refers to writing the various technical and technological styles mentioned above in English. Writing is a writing process, and the writer must grasp the characteristics of various styles and basic writing formats. To master the complete and correct format and content is the premise of listening, speaking, reading and writing.

\section{Features of Technical English}

Different from literary English and other forms of English, the contents and expressions of technical English as well as the words and sentences have their own characteristics. To sum up, there are two major points in total, namely, the characteristics of the words and the sentence structure of technical English.

In the expression of technical English, more intuitive, simple, accurate and unambiguous vocabularies are used.

Unlike everyday English or literary English, which has the perceptual image of thinking, technical English is not emotionally charged. Therefore, formal written words are often used instead of colloquial words or phrases of the same meaning. Therefore, in translation, these factors should be considered to enhance the logical relationship in the translation with concise terms and ensure that the translation is correct.

Logic of technical English

Technical English involves science and technology, technical equipment and testing process in science and technology. It generally describes and discusses objective things. It has strong objectivity, accuracy and rigor. Its language is standardized, logical and structured. Most of the description is the objective facts.

Target of technical English

Technical English translation is most used for technical and technological exchanges or to describe objective facts with clear purpose. Based on the features of technical English, the translation of technical English should follow corresponding translation standards. Technical English is to express and convey instructions and information, so its languages are accurate, objective and follows strict logic. In the translation of long sentences of technical English, on the basis on accurately conveying information, the translation should be fluent, concise and logic, which can also be deemed as the principle of translating long sentences in technical English.

\section{Development of Translating Technical English}

To further develop technical English translation studies, we must improve it from three aspects: basic research, practical research and theoretical research. In basic research, technical English translation should clarify and determine some basic concepts, improve the system architecture, and form a unified and complete theoretical discourse system.

In practical research, we should borrow more advanced research methods, such as corpus, to form a research method combining quantitative and qualitative analysis, summarize the translation strategies and methods that are suitable for the development of technical English translation, and improve our translation of technical English that "the translation is smooth but not acceptable" so as to improve the technical English translation communication effect in China. In the aspect of theoretical research, based on the existing theoretical research, technical English translation should further expand its theoretical perspective and form a systematic, deep-seated and interdisciplinary "translation" theory so as to serve the national strategy of "going global" that serves the Chinese culture.

The studies of technical English translation should take into consideration of not only the micro-language conversion, but also the macro social, historical and cultural context. It should not only elaborate on the systematic theories, but also improve the practical effect of technical English translation, serving for the national strategy of making the Chinese culture "go out". With the development of science and technology, it can be applied to not only the literary criticism, but also the translation studies, and even the studies of non-literary technical English translation. 


\section{Translation of Technical English Vocabulary}

Like toe translation of other styles, the translation of technical English also lies in the accurate understanding of the original text, and then use some translation skills to convert the text into Chinese based on the understanding of the original text, among which the key is to accurately understand the original text. In terms of the translation standard, Yan Fu, a translator in the late Qing Dynasty, proposed the famous principle of "faithfulness, expressiveness and elegance". To put it simple, it means that the translation should be "faithful", "smooth" and "elegant". To be more specific, the "translated version should be faithful to the original ideas", "the translated version should conform to the national standardized language", and "the translation version should keep the style of the original text" (Peng Zhuo-wu). Nida then put forward "functional equivalence", which means the translated version should keep the same with the original version in terms of lexical meaning, stylistic features and so on. For the translation of technical English, the translation should be loyal, clear, smooth and concise. There are two difficulties in understanding and translating technical English: one is vocabulary and the other is structure.

In terms of vocabulary, the frequency of professional vocabulary in scientific and technological documents is low, and there are more sub-technical words while functional words appear with the highest frequency. Relatively speaking, professional vocabularies have one meaning with few polysemy. However, when new words appear, the translators should know how to use the word formation to understand the meaning of the word and translate it correctly. In fact, it is not the professional vocabulary that is the most difficult to be understood in technical English, but some semi-technical verbs, adverbs and adjectives, particularly some phrasal verbs. This depends on the translator's understanding of the meaning of each word in polysemy and the context and the translator should also use appropriate professional knowledge to judge the word meaning.

\section{Application of Translated Technical Vocabulary}

In scientific articles, the meaning of one word is often specialized, i.e., one English word is not only used in various professional fields, and it contains different meanings.

Abbreviations are often used in technical English. With the rapid development of modern science, the number of abbreviations will increase day by day. An abbreviation often represents dozens of meanings.

There are three ways to construct acronyms in technical English:

The word part is truncated to form a new word, such as parachute-chute (parachute).

Add the first letter of each word in the phrase together to form new words, i.e., acronyms, such as Unidentified Flying Object-UFO.

Extract some letters from two words to form new words, such as telegram exchange-telex (telex). Use nouns and nouns extensively. English uses nouns more widely than Chinese and this phenomenon is more pronounced in technical English.

For example, in technical articles, "we can improve its performance by using superheated steam" shall be replaced by "an improvement of its performance can be achieved by the use of superheated steam". In order to express a certain concept or thing briefly and clearly, noun phrases are also widely used in technical English, such as energy lose, a day and night weather observation station.

\section{Conclusion}

With the rapid development of technologies, various new disciplines and fields emerge as the times require, and a large number of new technical and technological terms have emerged, especially the use of long sentences in English. This makes translation of technical and technical English documents more and more difficult. Therefore, on the one hand, we must constantly learn and master the skills of translating technical English literature. On the other hand, we must constantly improve our English and Chinese proficiency, strive to broaden our knowledge base and improve our own quality so as to achieve Chinese is good and rich in professional knowledge so that the translation level so as to continuously improve the 
translation of technical English documents. In short, when translating technical and technical articles, according to the language characteristics of technical English, it should be ensured that the translation is faithful to the original, smooth and fluent and the translators should master the basic knowledge of English and Chinese and some theoretical knowledge of translation, broaden the knowledge ken and develop a rigorous working attitude. Only in this way can we translate the science and technology documents more accurately.

\section{References}

[1] Torres-Martínez S. An English translation of the poem Schwarze Flocken ('Black Snowflakes') by Paul Celan[J]. 2015.

[2] Zhou F H. An Analysis of the Reproduction of XU Yuanchong's"Three Beauties"Theory in C-E Translation of Tang Poetry[J]. Journal of Qinzhou University, 2015.

[3] Yang M, Zha J M. Three Specious Directions of "Emulation Method" in English-Chinese Metrical Poetry Translation[J]. Journal of Nanchang Normal University, 2016.

[4] Wang Y M. Analyze on the Use and Translation of Numerals in Chinese Poetry[J]. Overseas English, 2015.

[5] Zou X J. Conceptual Blending of the Common Image Combination in Lovesick Poetry and Translation Strategy[J]. Journal of Hebei University of Technology, 2015.

[6] Alsaeed N H Q. Irish Poetry and Its Contribution to European Literature[J]. 2015, 5(4):27.

[7] Thangam R. A study on translation of modern prose and poetry from tamil to english[J]. Coimbatore, 2015.

[8] Lukes A. Mallarmé's Madness: Poetry, Pedagogy, and Translation[J]. Modernism/modernity, 2017, 24(1):141-160.

[9] Pusztaivarga I. Cultural Dimensions of Poetry Translation[J]. Acta Universitatis Sapientiae Philologica, 2017, 8(3).

[10]Huang Y J. Semantic Fuzziness of Quantifiers and its Analysis in Translating Ancient Chinese Poems into English[J]. Journal of Xihua University, 2015.

[11]Rudolf G I. The Translation of German Hymns into Romanian Between Poetry and Pragmatism[J]. Review of Ecumenical Studies Sibiu, 2016, 8(1):85-99.

[12] Li Q, Wu Y. Translation of Ancient Chinese Poetry in View of Iconicity Translation Theory:Case Study of Li Yu's Poetry[J]. Journal of Shenyang University, 2017. 